\title{
Esthesioneuroblastoma and Olfactory Preservation: Is it Reasonable to Attempt Smell Preservation?
}

\author{
Jamie J. Van Gompel ${ }^{1,2}$ Jeffrey R. Janus ${ }^{2}$ Joshua D. Hughes ${ }^{1}$ Janalee K. Stokken ${ }^{2}$ Eric J. Moore ${ }^{2}$ \\ Tarek Ryan ${ }^{1}$ Daniel L. Price ${ }^{2}$ Michael J. Link ${ }^{1,2}$

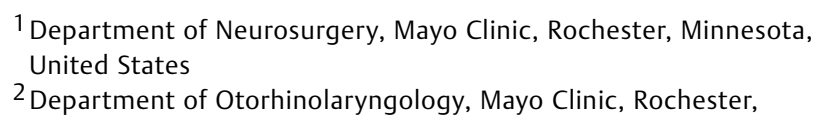

${ }^{2}$ Department of Otorhinolaryngology, Mayo Clinic, Rochester, \\ Minnesota, United States \\ Address for correspondence Dr. Jamie J. Van Gompel, MD, \\ Departments of Neurosurgery and Otorhinolaryngology, Mayo Clinic, \\ 200 First Street SW Rochester, MN 55905, United States \\ (e-mail: vangompel.jamie@mayo.edu).
}

J Neurol Surg B 2018;79:184-188.

\begin{abstract}
Keywords

- esthesioneuroblastoma

- olfactory neuroblastoma

- outcome

- olfactory nerve

- surgical treatment

Objective Olfactory preservation after resection of esthesioneuroblastoma (ENB) has been reported, however, the ability to predict tumor involvement of the olfactory system is critical to this surgical strategy. This study aims to answer the question: Can a surgeon predict, based on preoperative imaging, whether there is unilateral involvement of the olfactory system allowing for safe attempt of olfactory preservation? Methods This is a retrospective review of post-resection ENB meeting inclusion criteria of having bilateral olfactory tracts and bulbs submitted at the time of primary resection for pathologic margins. Five board-certified skull base surgeons blinded to the pathology individually reviewed the preoperative MRI scans to predict degree of tumor involvement.

Results Olfactory bulb involvement occurred in both bulbs in $35 \%$ of cases and unilateral in $39 \%$ of cases, and there was no involvement in $26 \%$ of cases sampled. When comparing physician prediction of involved tracts or bulbs, involvement was appropriate or over-called (i.e., called positive when pathology was in fact negative) in $96 \%$ of cases.

Conclusion This study demonstrates unilateral or no pathologic olfactory involvement of the olfactory system in $65 \%$ of cases. Our ability to predict this involvement, which may allow for a management strategy that attempts to preserve olfactory function, was accurate at $96 \%$. Therefore, interpretation of imaging and proceeding with smell preservation in ENB appears reasonable in this cohort.

Level of Evidence: Level $2 b$.
\end{abstract}

\section{Introduction}

Esthesioneuroblastoma (ENB) is a malignant neoplasm of the skull base that up until recently has been subject to aggressive en bloc or piece meal margin negative resection, resulting in good overall long term outcomes. ${ }^{1-4}$ Endoscopic approaches

received

April 23, 2017

accepted after revision

July 26, 2017

published online

August 28, 2017 (although with similar ultimate margin negative resections) have recently brought into question the necessity of these more extensive wide margin resections with emphasis on the patient's quality of life, particularly given the long-term survival of many ENB patients. ${ }^{5,6}$ In the past, we as a multidisciplinary treatment group adopted the practice of orbital

(c) 2018 Georg Thieme Verlag KG Stuttgart · New York
DOI https://doi.org/ 10.1055/s-0037-1606307. ISSN 2193-6331. 
preservation despite periorbital involvement, as these patients tend to do well and are spared from the morbidity and stigmata of orbital exenteration. ${ }^{7-9}$ It should be stated, however, that preservation of function is an important consideration, but should not compromise a margin negative resection in patients with ENB. ${ }^{1,10}$ Recently, authors have begun to report olfactory preservation for ENB as technically feasible, with postoperative imaging demonstrating complete resection in highly selected cases. ${ }^{11,12}$ Though challenging, olfactory preservation is possible, as reported in $14 \%$ of patients across a multi-institutional study that retained useful postoperative smell as objectively measured in the University of Pennsylvania Smell Identification Test (UPSIT). ${ }^{11}$ Although the surgical approach has been described, we do not understand the frequency of olfactory nerve or bulb involvement in those sampled, or is it known if imaging can predict unilateral, bilateral, or lack of involvement of tumor with respect to these structures. Therefore, in this series of pathologically sampled patients in which olfactory preservation was not attempted, we present data to support the use of preoperative imaging to predict potential candidates for smell preservation. This study aims to answer the question: Can a surgeon predict whether there is involvement of the olfactory bulbs or tracts based on preoperative imaging, allowing for safe attempt of olfactory preservation?

\section{Materials and Methods/Case Material}

Between January of 2000 and December 2016, 54 surgeries for either primary or recurrent ENB took place at Mayo Clinic, Rochester. This registry was retrospectively screened for cases who underwent craniofacial resection, which consisted either of endoscopic alone, subfrontal craniectomy, or bifrontal craniotomy with endoscopic assistance for primary resection of patients who underwent bilateral olfactory bulb and nerve sampling. Further, cases of Kadish D disease and incomplete data pertaining to olfactory bulb or nerve sampling (i.e., large bifrontal tumors in which the nerves were eroded or destroyed as part of the same) were excluded. This allowed for 26 patients for analysis.

The senior authors, blinded to the pathologically determined olfactory involvement, retrospectively reviewed the preoperative imaging and provided their opinions about whether there would be unilateral (right or left) or bilateral tumor involvement of the olfactory bulb or tract. This data was compared with the pathologic margins for analysis; imaging was available in 23 of the 26 cases for analysis. A case example of olfactory nerve and functional preservation is also presented, with objective smell testing at 1 year; this case was performed outside of the cases analyzed in this series.

\section{Results}

Overall, olfactory tract involvement occurred bilaterally in $26 \%$ of cases, unilaterally in $13 \%$ of cases, and had no involvement in $61 \%$ as confirmed on pathology (-Table 1 ). Olfactory bulb involvement occurred in both bulbs in $35 \%$ of cases and was unilateral in $39 \%$ of cases, and there was no involvement in $26 \%$
Table 1 Pathologic involvement of the olfactory system after primary resection for esthesioneuroblastoma

\begin{tabular}{|l|l|l|l|}
\hline Involvement & Unilateral & Bilateral & None \\
\hline Olfactory bulb/fila & $39 \%$ & $35 \%$ & $26 \%$ \\
\hline Olfactory tract & $13 \%$ & $26 \%$ & $61 \%$ \\
\hline Overall & $50 \%$ & $35 \%$ & $15 \%$ \\
\hline
\end{tabular}

of cases sampled. Overall, there was pathologic involvement of the olfactory bulb or tract in $85 \%$ of cases ( - Table 1 ), with involvement less than bilateral in almost two-thirds (65\%) of cases. When comparing physician prediction of involved tracts or bulbs, involvement was appropriate or over-called (i.e., called positive when pathology was in fact negative) in $96 \%$ of cases (-Table 2). Only one case demonstrated bilateral involvement, but was under-called by three surgeons as unilateral involvement ( $4 \%$ of cases).

\section{Case Example}

A 61-year-old male presented with recurrent sinus infections confined to the left nostril for 6 months. He underwent a sinus computed tomography (CT) examination in May 2015, which revealed nasal polyps in the left ethmoid region; no preoperative magnetic resonance imaging (MRI) was performed. Postoperative pathology after resection on demonstrated ENB was consistent with a low-grade Hyams pathology (grade 2). He underwent a positron emission tomography (PET) scan at that time, which was negative for metastases or regional lymph node involvement. He was advised that this was likely not a complete resection and was advised to seek further consultation. He presented with an MRI demonstrating likely residual disease at the skull base (-Fig. 1). The left olfactory nerve appeared to enhance minimally. The residual mass was judged to be a unilateral (left) Kadish B tumor. He was taken for an endoscopic, transnasal margin negative resection. A right-sided nasoseptal flap was fashioned. Residual disease was found in the superior left-sided septum and superior ethmoids; the left olfactory tract and bulb were resected and were negative on final pathology. Final microscopic margins were negative. The right olfactory apparatus and right septal olfactory epithelium were spared (-Fig. 1b). There were no postoperative complications. He had been followed up for 15 months without evidence of recurrence on MRI and endoscopic examination; he did not undergo postoperative radiation. The UPSIT was performed at 1 year; a score of a 32 of 40 was validated, which for male patients of 60 to 64 years of age is considered on the border of mild microsomia and normosmia demonstrating retained olfactory function.

\section{Discussion}

Although the situation in which one is confronted with the possibility of preserving olfaction is uncommon in esthesioneuroblastoma, as we become better at understanding of this 
Table 2 Cases evaluated by surgeons

\begin{tabular}{|c|c|c|c|c|c|c|}
\hline Case & Final pathologic & & & Surgeon $r$ & & \\
\hline & No involvement & Unilateral & Bilateral & Unilateral & Bilateral & Under-called $^{c}$ \\
\hline 1 & & & $x$ & 0 & 5 & \\
\hline 2 & $x$ & & & 5 & 0 & \\
\hline 3 & $x$ & & & 4 & 1 & \\
\hline 4 & & $x$ & $x$ & 0 & 5 & \\
\hline 5 & & $x$ & & 1 & 4 & \\
\hline 6 & $x$ & & & 5 & 0 & \\
\hline 7 & & & $x$ & 3 & 2 & $x$ \\
\hline 8 & $x$ & & & 4 & 1 & \\
\hline 9 & & & $x$ & 0 & 5 & \\
\hline 10 & & & $x$ & 0 & 5 & \\
\hline 11 & & $x$ & & 5 & 0 & \\
\hline 12 & & $x$ & & 0 & 5 & \\
\hline 13 & & $x$ & & 0 & 5 & \\
\hline 14 & & $x$ & & 2 & 3 & \\
\hline 15 & & $x$ & & 4 & 1 & \\
\hline 16 & & & $x$ & 0 & 5 & \\
\hline 17 & & $x$ & & 5 & 0 & \\
\hline 18 & & $x$ & & 0 & 5 & \\
\hline 19 & & & $x$ & 0 & 5 & \\
\hline 20 & & $x$ & & 5 & 0 & \\
\hline 21 & & & $x$ & 0 & 5 & \\
\hline 22 & & & $x$ & 0 & 5 & \\
\hline 23 & & $x$ & & 5 & 0 & \\
\hline
\end{tabular}

${ }^{a}$ Final pathologic margin represents actual pathologic involvement.

${ }^{\text {b}}$ Surgeon response is the number of surgeons calling unilateral or bilateral involvement.

'This column marks the under-called case.

disease, skull base anatomy, and the details of the endoscopic approach and reconstruction, we must consider preservation of one of the essences of life: sense of smell. ${ }^{13}$ You need look no further than poetry with vivid descriptions of the scent of a rose or putrid smell of death to see the important emotional role and impact on memory that olfaction has over the other senses. Beyond this, those with impaired olfaction are at higher risk of being injured in a fire (cannot smell smoke or gas leak) or suffer from food poisoning (unable to tell if something is spoiled). ${ }^{14,15}$

As demonstrated in the case report attached to this report, there were four patients with normal or mildly reduced olfaction after resection of an ENB, demonstrating the feasibility of an olfactory preserving technique. Wessell et al reported the first case in the literature available in 2014 in a patient undergoing unilateral en bloc resection of an ENB with postoperative mild olfactory impairment. ${ }^{12}$ Tajudeen et al reported a multi-institutional series where 14 patients underwent unilateral olfactory bulb or tract resection; of these six had some evidence of olfactory loss. ${ }^{11}$ Moreover, two had normal or near normal olfaction. ${ }^{11}$ Therefore, although technically feasible, our report and others acknowledge that only time will tell whether equivalent long-term outcome is seen in this cohort of patients. In this authors' view, we only attempt smell preservation in biopsy-proven low Hyams grade ENB with limited cribriform involvement and no distant disease (i.e., Kadish A, B, and some limited C patients).

Although preservation of olfaction is selectively possible, the issue of course addressed with this paper is that is it responsible or reasonable. Here, we demonstrate with good preoperative imaging and staging that surgeons can reliably predict what margins need to be resected. Having five surgeons evaluate in a blinded fashion whether none, unilateral (left or right), or both olfactory bulbs and tracts are involved leads to calling correctly all cases, but one. Therefore, 96\% of cases were interpreted correctly, with one case having bilateral olfactory bulb involvement called as unilateral by three of five surgeons. We recommend a high quality skull base CT and coronal T2-weighted MRI in addition to a coronal T1 with gadolinium examination to determine the extent of involvement. While this demonstrates that interpretive error is possible, it certainly validates a strategy to attempt to preserve 


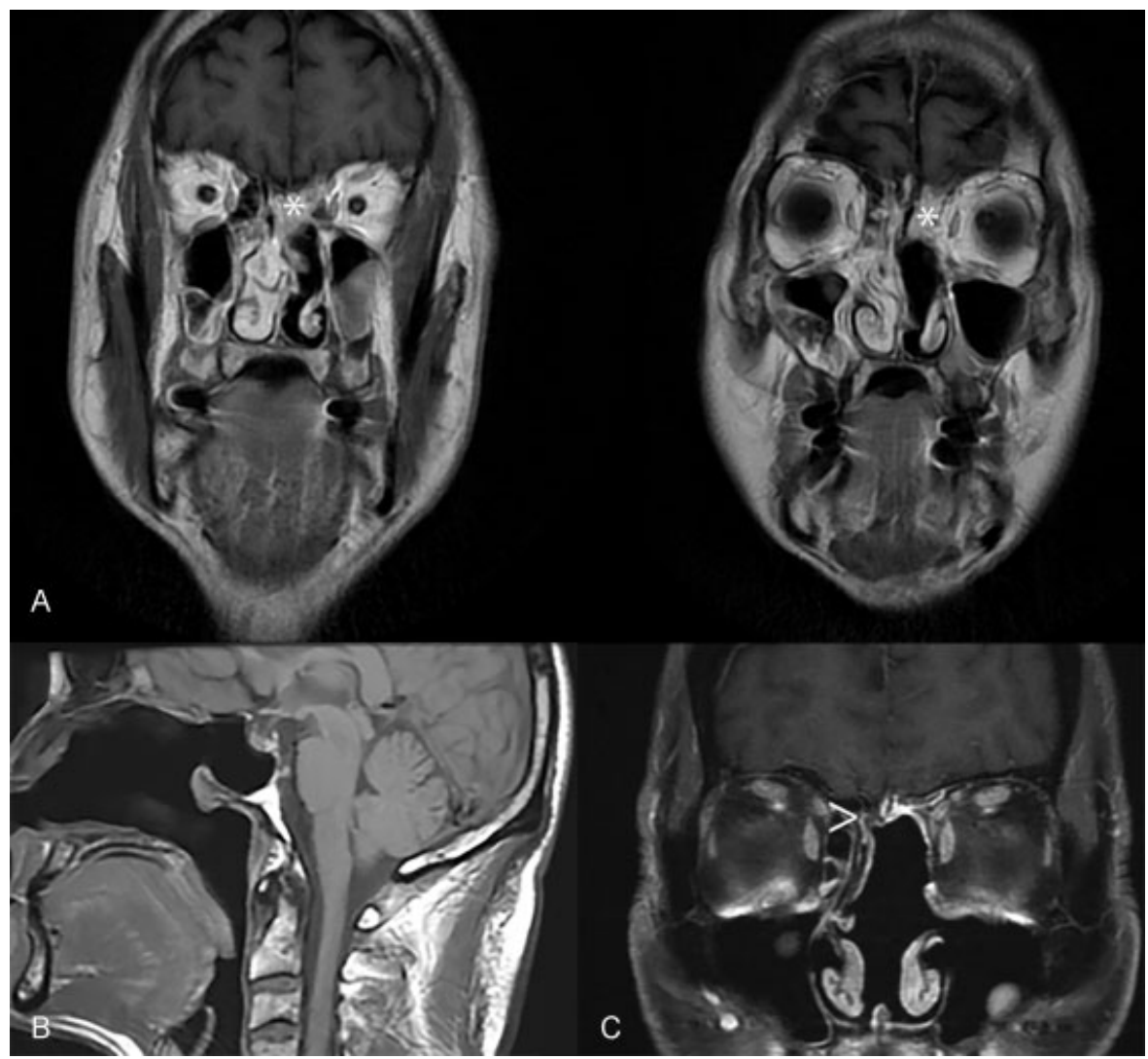

Fig. 1 (A) Coronal MRI T1 with gadolinium demonstrating the extent of residual disease $\left({ }^{*}\right)$ prior to margin negative resection of this low-grade esthesioneuroblastoma. (B) Sagittal flair MRI demonstrating postoperative resection and flap placement of ENB. (C) Coronal T1 MRI with gadolinium MRI demonstrating persevered olfactory system ( $>$ ) and adjacent nasoseptal flap placement. ENB, esthesioneuroblastoma; MRI, magnetic resonance imaging.

olfaction based on preoperative imaging given the accuracy seen in this pathologically confirmed cohort.

Recognizing the limits of pathology here, in that although the pathology may be negative on final analysis, this does not assume that all portions of the nerve or bulb were sent for final analysis. Therefore, although a negative nerve margin demonstrates the portion that is sent is not involved by tumor, there may be portions more distal that may have been positive and not included. In fact, given the presumed origin of ENB, we suspect distal olfactory fila to be definitively involved although unseen on pathology due to their destruction by the tumor. Further, inadequate surgical sampling to preserve olfaction may lead to an inappropriate margin and predispose the patient to recurrence of their cancer. Acknowledging these limitations, we observed pathologic involvement of the olfactory bulbs or tracts in $85 \%$ of cases, with unilateral or no involvement in $53 \%$ of cases.

\section{Conclusions}

This study demonstrates unilateral or no pathologic olfactory involvement of the olfactory bulbs or tracts in $65 \%$ of cases. Our ability to predict this involvement, which would allow for a management strategy that attempts to preserve olfac- tory function, was accurate at $96 \%$. Therefore, interpretation of imaging and proceeding with smell preservation in ENB appears reasonable in this cohort.

\section{Disclosure \\ None.}

Conflicts of Interest

None.

Acknowledgments

None.

\section{References}

1 Dulguerov P, Calcaterra T. Esthesioneuroblastoma: the UCLA experience 1970-1990. Laryngoscope 1992;102(08):843-849

2 Kadish S, Goodman M, Wang CC. Olfactory neuroblastoma. A clinical analysis of 17 cases. Cancer 1976;37(03):1571-1576

3 Kane AJ, Sughrue ME, Rutkowski MJ, et al. Posttreatment prognosis of patients with esthesioneuroblastoma. J Neurosurg 2010; 113(02):340-351

4 Van Gompel JJ, Giannini C, Olsen KD, et al. Long-term outcome of esthesioneuroblastoma: hyams grade predicts patient survival. J Neurol Surg B Skull Base 2012;73(05):331-336

5 Manthuruthil C, Lewis J, McLean C, Batra PS, Barnett SL. Endoscopic endonasal management of olfactory neuroblastoma: a 
retrospective analysis of 10 patients with quality-of-life measures. World Neurosurg 2016;90:1-5

6 Unger F, Haselsberger K, Walch C, Stammberger H, Papaefthymiou G. Combined endoscopic surgery and radiosurgery as treatment modality for olfactory neuroblastoma (esthesioneuroblastoma). Acta Neurochir(Wien) 2005;147(06):595-601, discussion 601-602

7 Essig GF, Newman SA, Levine PA. Sparing the eye in craniofacial surgery for superior nasal vault malignant neoplasms: analysis of benefit. Arch Facial Plast Surg 2007;9(06):406-411

8 Herr MW, Gray ST, Erman AB, Curry WT, Deschler DG, Lin DT. Orbital preservation in patients with esthesioneuroblastoma. J Neurol Surg B Skull Base 2013;74(03):142-145

9 McCary WS, Levine PA, Cantrell RW. Preservation of the eye in the treatment of sinonasal malignant neoplasms with orbital involvement. A confirmation of the original treatise. Arch Otolaryngol Head Neck Surg 1996;122(06):657-659

10 Levine PA. Would Dr. Ogura approve of endoscopic resection of esthesioneuroblastomas? An analysis of endoscopic resection data versus that of craniofacial resection. Laryngoscope 2009; 119(01):3-7

11 Tajudeen BA, Adappa ND, Kuan EC, et al. Smell preservation following endoscopic unilateral resection of esthesioneuroblastoma: a multi-institutional experience. Int Forum Allergy Rhinol 2016;6(10):1047-1050

12 Wessell A, Singh A, Litvack Z. Preservation of olfaction after unilateral endoscopic approach for resection of esthesioneuroblastoma. J Neurol Surg Rep 2014;75(01):e149-e153

13 Croy I, Nordin S, Hummel T. Olfactory disorders and quality of life-an updated review. Chem Senses 2014;39(03):185-194

14 Miwa T, Furukawa M, Tsukatani T, Costanzo RM, DiNardo LJ, Reiter ER. Impact of olfactory impairment on quality of life and disability. Arch Otolaryngol Head Neck Surg 2001;127(05): 497-503

15 Pence TS, Reiter ER, DiNardo LJ, Costanzo RM. Risk factors for hazardous events in olfactory-impaired patients. JAMA Otolaryngol Head Neck Surg 2014;140(10):951-955 